\title{
Increase of $Q$-factor of RF Magnetic Thin Film Inductor by Introducing Slit-patterned Magnetic thin film and Multi-line-conductor Spiral Coil
}

\author{
Takayuki Maruyama ${ }^{1}$, Yosuke Obinata ${ }^{1}$, Makoto Sonehara ${ }^{1}$, Kenji Ikeda $^{2}$, Toshiro Sato ${ }^{1}$ \\ ${ }^{1}$ Spin Device Technology Center, Shinshu University, Nagano 380-8553, Japan \\ ${ }^{2}$ Research and Development Center, TAIYO YUDEN CO., Takasaki 370-3347, Japan
}

\begin{abstract}
In order to increase $Q$-factor of RF magnetic thin film inductor integrated in Chip-size-package RF-ICs, two schemes have been investigated experimentally. For suppressing the in-plane eddy current of the magnetic thin film, a slit-patterned structure was introduced, and it was found that the slit-patterned magnetic thin film is very effective for increasing $Q$-factor. On the other hand, for suppressing ac copper loss, a multi-line-conductor spiral coil was introduced, and it was found that the multi-line coil is effective for improving decrease of inductance at high frequencies. By introducing the two schemes, a maximum $Q$-factor of 18 at $1.5 \mathrm{GHz}$ was obtained in a spiral inductor with a bottom slit-patterned $\mathrm{CoFeSiO} / \mathrm{SiO}_{2}$ granular multilayer film and triple-line-conductor spiral coil.
\end{abstract}

Index Terms-On-chip RF magnetic thin film devices, inductor, $Q$-factor, eddy current, ac copper loss, RF front/end, cell-phone, wireless LAN

\section{INTRODUCTION}

$\mathrm{R}^{\mathrm{E}}$ ECENTLY, various on-chip magnetic thin film inductors for RF applications were reviewed by D. S. Gardner et al.

[1]. In order to develop the RF magnetic thin film devices integrated in Chip-size-package RF-ICs, the authors have also fabricated a CoFeB magnetic thin film directional coupler [2], [3] and spiral inductor [4], [5].

However, the maximum $Q$-factor of the previously fabricated inductors was lower than 10 because of high frequency losses consisting of magnetic loss and copper loss. High frequency magnetic loss includes eddy current loss, and ferromagnetic resonance (FMR) loss near FMR frequency. Therefore magnetic thin film for RF applications should have the following features; higher electrical resistivity for suppressing eddy current, higher FMR frequency and narrow FMR linewidth for suppressing FMR loss.

K. Ikeda et al. [6] reported a $\mathrm{CoFeSiO} / \mathrm{SiO}_{2}$ granular multilayer film with a FMR frequency of $2.4 \mathrm{GHz}$ and very narrow FMR linewidth. However, the electrical resistivity of $\mathrm{CoFeSiO}$ granular layer was not so high (about $1 \mu \Omega \mathrm{m}$ ). Therefore when the granular multilayer film was used as a magnetic core of the spiral inductor, the in-plane eddy current would be induced in each $\mathrm{CoFeSiO}$ granular layer by perpendicular magnetic flux component in the spiral inductor. The perpendicular flux component also influences on spiral coil, that is, it gives rise to eddy current in each coil conductor and results in non-uniform current density distribution in the coil conductor. Non-uniform current density causes excess copper loss at high frequencies.

In this study, in order to increase the $Q$-factor of magnetic thin film inductor for RF applications, two schemes have been introduced for suppressing high frequency losses.

For suppressing the in-plane eddy current loss of the magnetic core, four kinds of slit-patterned magnetic film

Manuscript received February 21, 2009, revised XXXX, 2011.

Corresponding author: T. Sato (e-mail:sato@yslab.shinshu-u.ac.jp). structures have been introduced. K. Araki et al. [7] reported that such slit-patterned magnetic film structure contributes to obtain higher FMR frequency due to the increase of demagnetizing field effect. In this study, the authors focus on the effect of slit-patterned structure on the in-plane eddy current suppression.

For suppressing excess copper loss at high frequencies, multi-conductor-line spiral coil has been introduced. Such coil structure contributes to improve non-uniform current density distribution in the coil conductor. The same method for suppressing $a c$ copper loss was already confirmed in the magnetic film inductor for $\mathrm{MHz}$ switching micro DC-DC converter [8]. The authors have investigated whether this scheme is effective or not for suppressing ac copper loss of spiral inductor at RF band.

\section{Two SCHEMES FOR SuPPRESSING High FrEQUENCY LOSSES}

When the metallic magnetic thin film is used in spiral inductor as shown in Fig. 1(a), the perpendicular flux component $\phi_{p}$ gives rise to the eddy current in both magnetic film and coil conductor. In this study, two schemes have been introduced to suppress both eddy current losses.

The perpendicular flux component $\phi_{p}$ gives rise to the inplane eddy current in the magnetic thin film, which dominates over high frequency magnetic loss below FMR frequency. In this study, in order to suppress the in-plane eddy current, slitpatterned structure has been introduced to magnetic film as shown in Fig. 1(b).

On the other hand, also in each conductor line of the spiral coil, the perpendicular flux $\phi_{p}$ gives rise to eddy current in the conductor line, and the current density distribution becomes non-uniform. Dynamic loss resistance, $\delta R$ defined as $R_{a c}-R_{d c}$, when it is owing to non-uniform current density distribution only, can be expressed as follows [9], 


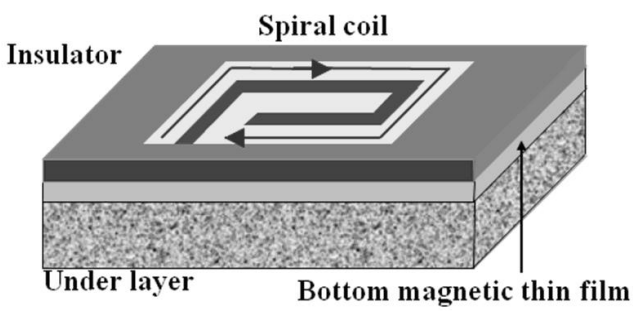

(a)Basic structure
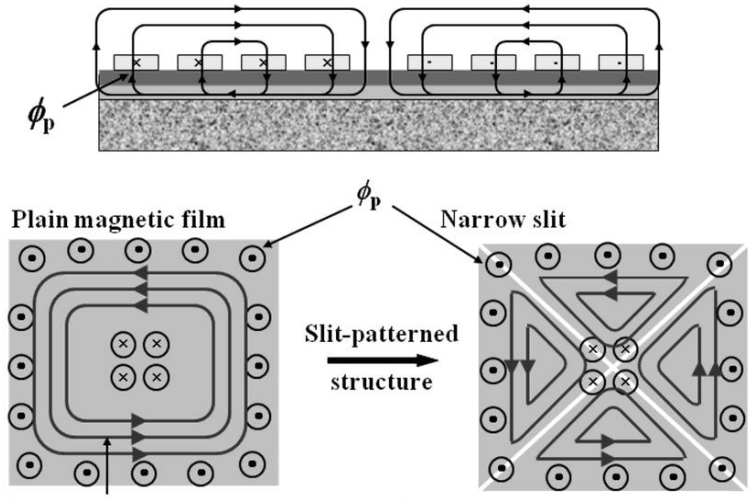

In-plane eddy current

Shortening of eddy current path

(b) Slit-patterned magnetic thin film
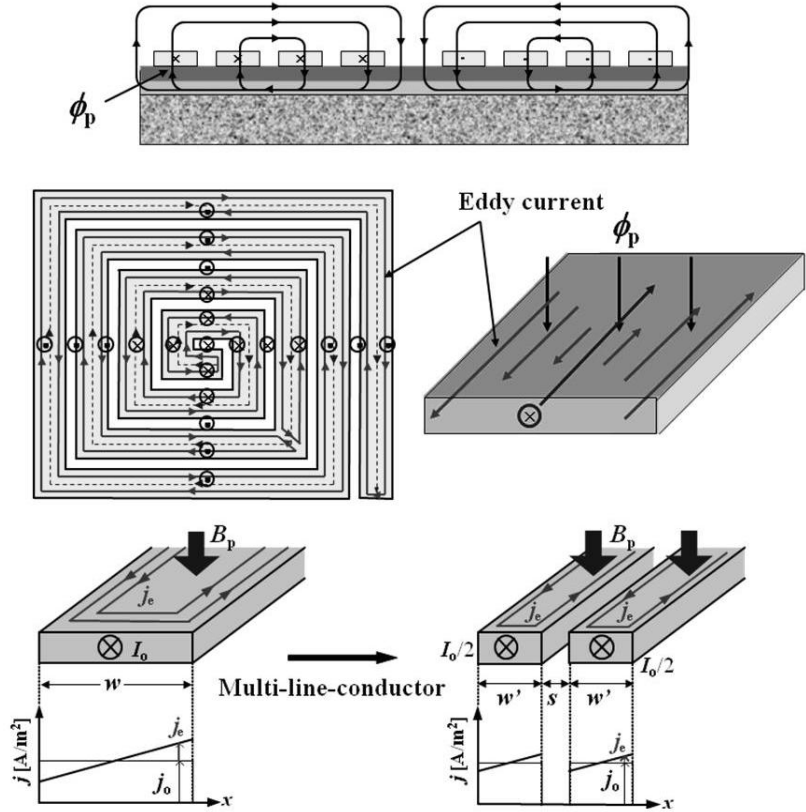

(c) Multi-line-conductor spiral coil

Fig. 1 Two schemes for suppressing high frequency losses for spiral inductor with a bottom metallic magnetic thin film.

$$
\delta R=K \frac{f^{2} t_{c} w^{3}}{12 \rho}
$$

where $f$ is the frequency, $t_{c}$ is the conductor thickness, $w$ is the conductor line width, $\rho$ is the electrical resistivity, and $K$ is a constant, respectively. Since $\delta R$ is proportional to $w^{3}$, in order to suppress the high frequency copper loss owing to nonuniform current density distribution, multi-conductor-line spiral coil structure has been introduced as shown in Fig. 1(c).

\section{MAGNETIC THIN FILM INDUCTOR UNDER INVESTIGATION}

\section{A. Inductor structure and fabrication method}

Fig. 2 shows the inductor structures for investigating the effect of two schemes on the increase of $Q$-factor. The fabricated inductors with $850 \times 850 \mu \mathrm{m}$ foot-print consisted of a 4-turn, $3 \mu \mathrm{m}$ thick copper spiral coil on the bottom $\mathrm{CoFeSiO} / \mathrm{SiO}_{2}$ granular multilayer film with an uniaxial magnetic anisotropy aligned in the horizontal direction. Therefore rotation magnetization is dominant in half area of the spiral inductor. The granular multilayer film made by ICP sputtering had a $6 \mathrm{~nm}-\mathrm{CoFeSiO} / 1 \mathrm{~nm}-\mathrm{SiO}_{2}$ structure and total CoFeSiO thickness of $100 \mathrm{~nm}$.

In this study, four kinds of the slit-patterned magnetic thin film structures (Type A to D in Fig. 2(b)) and three kinds of spiral-coil structures with a single, double and triple-line conductor (Fig. 2(c)) were introduced. Narrow $5 \mu \mathrm{m}$-width slits were introduced by photo-resist lift-off process for the magnetic film. Three kinds of 4-turn, $3 \mu \mathrm{m}$ thick copper spiral coils were made by electroplating method, and had a $3 \mu \mathrm{m}$ constant spacing between adjacent conductor lines.

\section{B. Magnetic properties of $\mathrm{CoFeSiO} / \mathrm{SiO}_{2}$ granular} multilayer film

Fig. 3 shows the magnetic properties of $\mathrm{CoFeSiO} / \mathrm{SiO}_{2}$

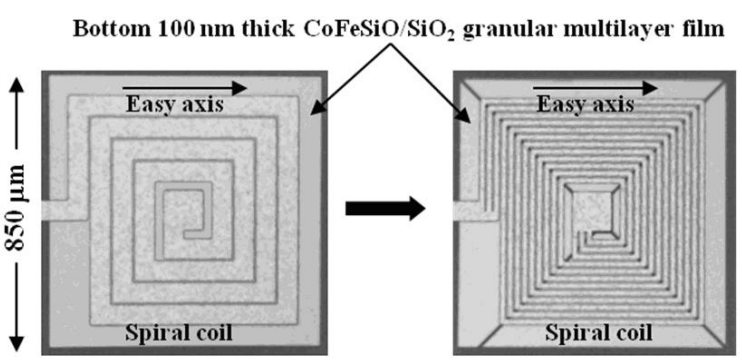

Single-line spiral coil Plain magnetic core

Triple-line spiral coil

Type A slit-patterned magnetic film

(a) Two examples of fabricated spiral inductor with a bottom 100 nm thick $\mathrm{CoFeSiO} / \mathrm{SiO}_{2}$ granular multilayer magnetic film

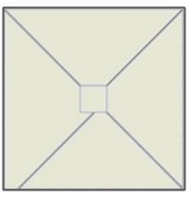

Type A

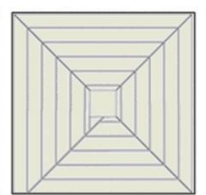

Type B

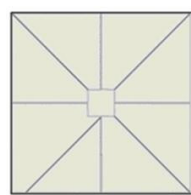

Type C

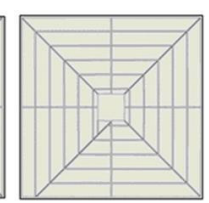

Type D (b) Four kinds of slit-patterned magnetic thin film structures
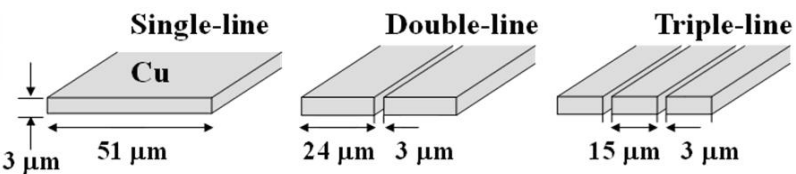

(c) Three kinds of multi-line-conductor spiral coils

Fig. 2 Structures of the fabricated magnetic thin film inductors for investigating the effect of two schemes on the increase of $Q$ factor. 


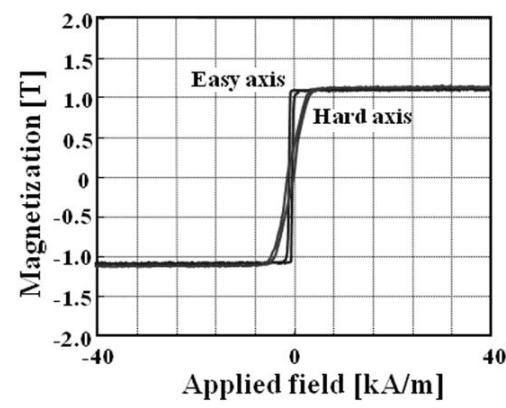

(a) Static magnetization curve

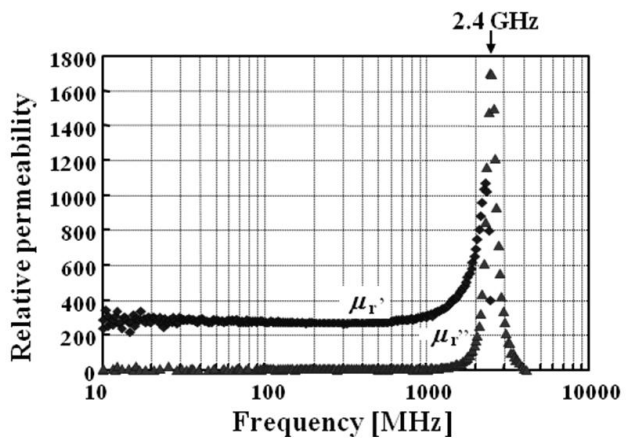

(b) Complex permeability v.s. frequency

Fig. 3 Magnetic properties of $\mathrm{CoFeSiO} / \mathrm{SiO}_{2}$ granular multilayer magnetic film with a $6 \mathrm{~nm}-\mathrm{CoFeSiO} / 1 \mathrm{~nm}-\mathrm{SiO}_{2}$ structure and total $\mathrm{CoFeSiO}$ thickness of $100 \mathrm{~nm}$.

granular multilayer magnetic film. The film had a saturation magnetization of $1.1 \mathrm{~T}$ which was diluted by a factor of 0.86 through the non-magnetic $\mathrm{SiO}_{2}$ layer. Anisotropy magnetic field $H_{k}$ was estimated to be $4 \mathrm{kA} / \mathrm{m}$ (50 Oe). The complex permeability v.s. frequency, which was measured by a thin film permeameter method [10], exhibited a FMR frequency of $2.4 \mathrm{GHz}$ and narrow FMR linewidth [6].

\section{RESUlTS AND DisCUSSION}

\section{A. Effect of two schemes on inductance and Q-factor}

Fig. 4 shows the experimental results of inductance $L$ and $Q$-factor v.s. frequency measured in various inductors, (a) is the effect of slit-patterned magnetic thin film structure with the same triple-line-conductor spiral coil, and (b) is the effect of multi-line-conductor spiral coil with the same Type A slitpatterned magnetic thin film.

As shown in Fig. 4(a), maximum $Q$-factor was increased by introducing the slit-patterned magnetic thin film. Especially, Type D consisting of fine magnetic film segments had the maximum $Q$-factor of about 18 at around $1.5 \mathrm{GHz}$. Since Type B and D inductor had slits aligned in the conductor line, FMR frequency of magnetic film segment was estimated to be higher than intrinsic value because of shape anisotropy (demagnetizing field). Therefore, high $Q$-factor obtained in Type B and D may be not only due to the in-plane eddy current suppression but also increase of FMR frequency.

From Fig. 4(b), the inductance of the single-line spiral inductor decreased with increasing frequency below $1 \mathrm{GHz}$. In case of such wide-line-conductor spiral coil, since the induced eddy current causes a decrease of magnetic flux linkage, it was considered that the inductance decreases with increasing frequency. On the other hand, multi-line spiral inductor had a constant inductance at least up to $1 \mathrm{GHz}$, and had higher maximum $Q$-factor than that of single-line spiral inductor because eddy current can be suppressed by narrow conductor line.

As shown in the prior art [11], the maximum $Q$-factor of the previously fabricated magnetic thin film inductor with microslits was lower than 10 . In our study, the $Q$-factor of about 18 was obtained by introducing the two schemes. We consider that the $Q$-factor of 18 is higher than that of any prior art.

\section{B. Effect of two schemes on dynamic loss resistance}

The measured dynamic loss resistance $\delta R$ is different from Eq. (1), because it depends not only on the ac copper loss but also on the high frequency magnetic loss.

Fig. 5 shows the frequency dependence of dynamic loss resistance, the left side figure is the effect of slit-patterned magnetic thin film structure with the same triple-lineconductor spiral coil, and the right side figure is the effect of multi-line-conductor spiral coil with the same Type A slitpatterned magnetic thin film.

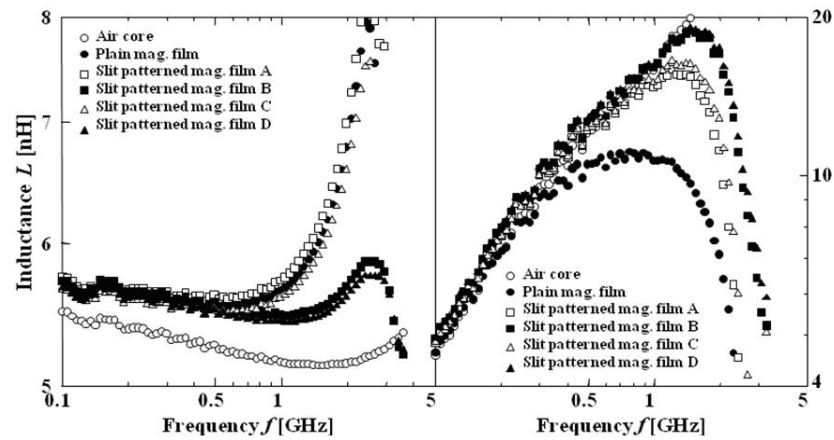

(a) Effect of slit-patterned magnetic thin film structure

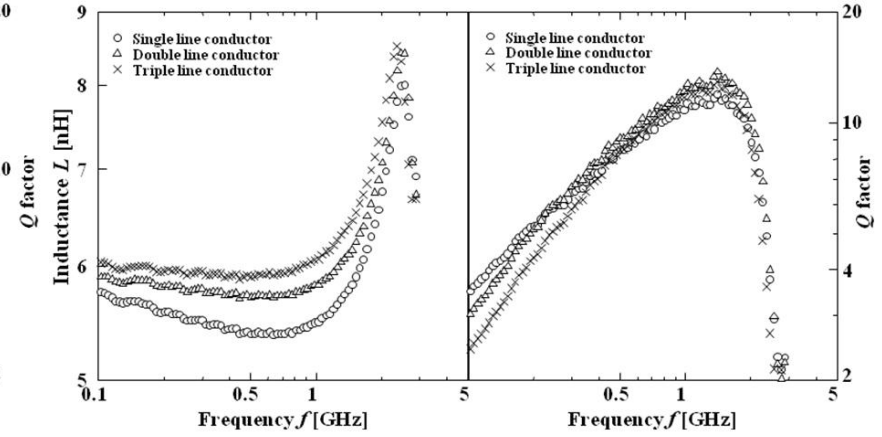

(b) Effect of multi-line-conductor spiral coil

Fig. 4 Effect of two schemes on inductance and $Q$-factor, (a) : effect of slit-patterned magnetic thin film structure with same triple-lineconductor spiral coil, (b) : effect of multi-line-conductor spiral coil with same Type A slit-patterned magnetic thin film. 

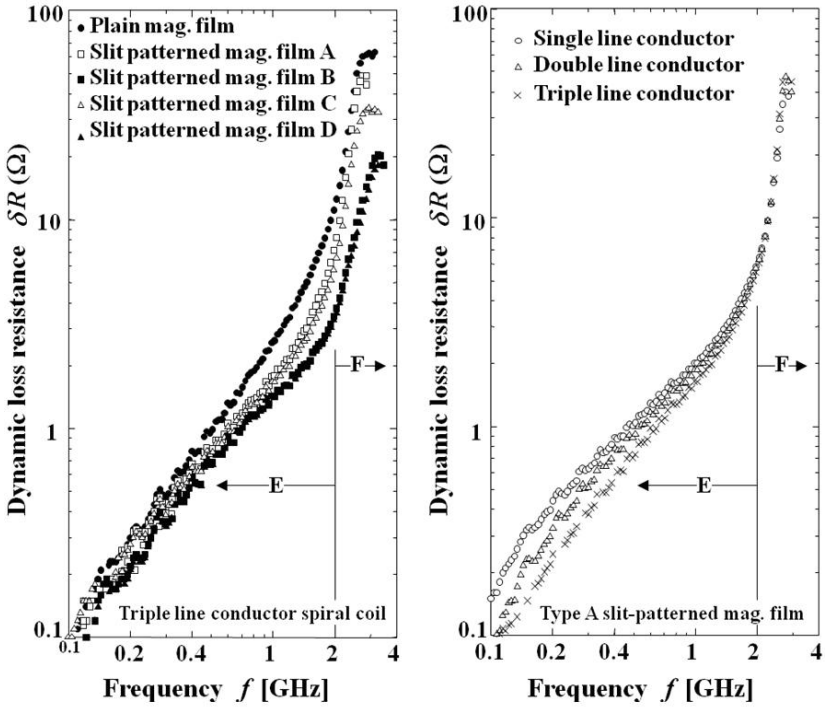

Fig. 5 Effect of two schemes on the decrease of dynamic loss resistance.

The $\delta R$ increased markedly at high frequencies over $2 \mathrm{GHz}$ (region $\mathrm{F}$ in Fig. 5). The authors considered that such behavior is owing to FMR loss of magnetic thin film. Since the Type B and D slit-patterned magnetic thin film was estimated to have higher FMR frequency due to shape anisotropy, $\mathrm{B}$ and $\mathrm{D}$ exhibited small $\delta R$ at $\mathrm{GHz}$ band. Therefore the slit-patterned magnetic thin film structure was effective for suppressing in-plane eddy current in the frequency region below $2 \mathrm{GHz}$ (region $\mathrm{E}$ in Fig. 5). On the other hand, as shown in the right side figure of Fig. 5, the effect of multi-line-conductor spiral coil was effective for decreasing $\delta R$ below $1 \mathrm{GHz}$.

From the experimental results, the dynamic loss resistance can be suppressed in the wide frequency range by introducing both slit-patterned magnetic thin film and multi-lineconductor spiral coil.

\section{CONCLUSION}

In order to increase the $Q$-factor of RF magnetic thin film inductor integrated in Chip-size-package RF-ICs, two schemes have been investigated experimentally using $\mathrm{CoFeSiO} / \mathrm{SiO}_{2}$ granular multilayer magnetic thin film and copper spiral coil. The slit-patterned fine segment magnetic thin film structure was effective not only for suppressing inplane eddy current but also for suppressing FMR loss due to increase of FMR frequency. The multi-line-conductor spiral coil was effective for suppressing $a c$ copper loss at lower frequency region below FMR frequency. Consequently, by introducing the two schemes, high frequency losses can be reduced at wide frequency range.

The fabricated spiral inductor with a slit-patterned fine segment magnetic thin film structure and triple-line-conductor spiral coil had a high $Q$-factor of 18 at $1.5 \mathrm{GHz}$.

\section{ACKNOWLEDGMENT}

This work was supported in part by a Grant-in-Aid for Scientific Research of Japan Society for the Promotion of Science (JSPS), No. 20560321.

\section{REFERENCES}

[1] D. S. Gardner, G. Schrom, F. Paillet, B. Jamieson, T. Karnik, and S. Borkar, "Review of On-Chip Inductor Structures with Magnetic Films", IEEE Transactions on Magnetics, Vol.45, No.10, pp.4760-4766 (2009).

[2] K. Takizawa, M. Nakazawa, T. Sato, K. Yamasawa, Y. Miura, M. Munakata, M. Yagi, "Directional Coupler Composed of CoFeB Metallic Magnetic Film/Polyimide Dielectric Film Hybrid Transmission-Line", Asia-Pacific Microwave Conference 2005 Proc., pp. 2819-2822, (2005).

[3] S. Mizuta, M. Nakazawa, K. Takizawa, T. Sato, K. Yamasawa, Y. Miura, Y. Miyake, M. Akie, Y. Uehara, M. Munakata, M. Yagi, "Large-signal Transmission Characteristics of a $\mathrm{CoFeB}$ Magnetic Thin Film Directional Coupler for Cellular Phones", Journal of the Magnetics Society of Japan, Vol.32, No.3, pp.376-381, (2008). (in Japanese)

[4] T. Maruyama, M. Yuki, D. Kobayashi, T. Minemura, M. Sonehara, K. Ikeda, T. Sato, "CoFeSiO/SiO ${ }_{2}$ Magnetic Thin Film Inductor for CSP RF-IC", 2010 International Conference on Microwave Magnetics in Boston, A2-5, June 2010.

[5] K. Ikeda, T. Suzuki, T. Maruyama, T. Minemura, M. Sonehara, and T. Sato, "Double spiral inductors using $\mathrm{CoFeSiO} / \mathrm{SiO}_{2}$ multilayer granular films with very narrow ferromagnetic resonance bandwidth", Journal of the Magnetics Society of Japan, Vol.34, No.2, pp.123-130 (2010). (in Japanese)

[6] K. Ikeda, T. Suzuki, T. Sato, $\mathrm{CoFeSiO} / \mathrm{SiO}_{2}$ multilayer granular films with very narrow ferromagnetic resonant linewidth, IEEE Transactions on Magnetics, Vol.45, No.10, pp.4290-4293 (2009).

[7] K. Araki, M. Munakata, M. Namikawa, M. Yagi, and Y. Shimada, "Magnetic Properties and Anisotropy Field of Slit-Patterned CoFe Films with High Saturation Magnetization", Journal of the Magnetics Society of Japan, Vol.28, No.9, pp.997-1000 (2004). (in Japanese)

[8] T. Sato, K. Yamasawa, H. Tomita, T. Inoue, T. Mizoguchi, "FeCoBN magnetic thin film inductor for $\mathrm{MHz}$ switching micro DC-DC converters", IEEJ Transactions on Industrial Applications, Vol.121-D, No.1, pp.84-89 (2001).

[9] T. Sato, K. Yamasawa, H. Tomita, T. Inoue, T. Mizoguchi, "Planar power inductor using $\mathrm{FeCoBN}$ magnetic film with high saturation magnetization and high electrical resistivity", Proc. of the International Power Electronics Conference in Tokyo (IPEC-Tokyo2000), pp.303-308, (2000).

[10] M. Yamaguchi, Y. Miyazawa, and K.-I. Arai, "A New 1 MHz-9 GHz Thin-Film Permeameter Using a Side-Open TEM Cell and a Planar Shielded-Loop Coil", Transactions of the Magnetics Society of Japan, Vol. 3, No. 4, pp. 137-140 (2003).

[11] M. Yamaguchi, M. Baba, K. Suezawa, T. Moizumi, K. I. Arai, A. Haga, Y. Shimada, S. Tanabe, and K. Itoh, "Improved RF integrated magnetic thin-film inductors by means of micro slits and surface planarization techniques", IEEE Transactions on Magnetics, Vol. 36, No. 5, pp. 34953498, (2000) 